\title{
Espondilite tuberculosa: uma revisão de 31 pacientes do Hospital Santa Marcelina
}

\author{
Tuberculous spondylitis: a report of thirty one cases from \\ Santa Marcelina Hospital
}

\section{Espondilitis tuberculosa: una revisión de 31 pacientes del Hospital Santa Marcelina}

\author{
Luiz Cláudio Lacerda Rodrigues ${ }^{1}$ \\ Adalberto Bortoletto ${ }^{2}$ \\ Marcelo Hide Matsumoto ${ }^{3}$
}

\section{RESUMO}

Introdução: a infecção da coluna vertebral pelo bacilo de Koch costuma ser devastadora, sendo necessários seu diagnóstico e tratamento precoce. Objetivo: avaliar o tratamento e o seguimento em relação à dor, cifose residual, imagem por ressonância magnética (RM), e a importância da biópsia correlacionando-a com o tratamento clínico. Métodos: estudo retrospectivo de 31 pacientes com diagnóstico de espondilite tuberculosa, fazendo uma análise estatística dos dados descritivos: sexo, idade, status neurológico, segmento vertebral acometido, presença de abscesso e cifose residual, e suas correlações clínicas importantes comparando nossos casos com a literatura e correlacionando seus dados, tais como sexo e faixa etária mais comum, se a presença do abscesso influencia no déficit neurológico ou na cifose residual. Resultados: a amostra identificou uma incidência em 23 homens e 8 mulheres. Foi identificado abscesso frio em 4 pacientes, sendo os que apresenta-

\section{ABSTRACT}

Introduction: the infection of the spine by the mycobacterium tuberculosis is often devastating, requiring early diagnosis and treatment. Objective: to assess the treatment and follow-up regarding the pain, residual kyphosis, image of magnetic resonance imaging (MRI), and importance of biopsy relating to the clinical treatment. Methods: retrospective study of 31 patients with diagnosis tuberculous spondylitis making a statistical analysis, studying the data descrition: gender, age, neurological status, spinal segment and kyphosis abscess, presence of residual kyphosis and their clinical correlations, comparing our major cases with literature and relashionship, and if the presence of abscess can influence in on neurological deficit or residual kyphosis. Results: the sample identified an incidence in 23 men and 8 women; cold abscess was identified in 4 patients, and how those with a severe deformity final percutaneous biopsy was performed in 19 patients

\section{RESUMEN}

Introducción: la infección de la columna vertebral por el bacilo de Koch acostumbra ser devastadora, siendo necesario el diagnóstico y tratamiento precoz. Objetivo: evaluar el tratamiento y el seguimiento en relación al dolor, cifosis residual, imagen en la resonancia magnética (RM) y la importancia de la biopsia correlacionándola con el tratamiento clínico. Métodos: estudio retrospectivo de 31 pacientes con diagnóstico de espondilitis tuberculosa haciendo un análisis estadístico de los datos descriptivos: sexo, edad, estatus neurológico, segmento vertebral comprometido, presencia de absceso y cifosis residual, así como sus correlaciones clínicas importantes comparando nuestros casos con la literatura y correlacionando sus datos con lo referente al sexo y la tasa etaria donde fue más común, si la presencia del absceso influyó en el déficit neurológico o en la cifosis residual. Resultados: la muestra identificó una incidencia en 23 hombres y 8 mujeres. Fue identificado absceso frío en cuatro pacientes,

\footnotetext{
Trabalho realizado no setor de Ortopedia e Traumatologia do Hospital Santa Marcelina - HSM - São Paulo (SP), Brasil.

'Médico assistente do Serviço de Patologias da Coluna Vertebral do Hospital Santa Marcelina - HSM - São Paulo (SP), Brasil.

${ }^{2}$ Médico chefe do Serviço de Patologias da Coluna Vertebral do Hospital Santa Marcelina - HSM - São Paulo (SP), Brasil.

${ }^{3}$ Chefe do Serviço de Ortopedia e Traumatologia do Hospital Santa Marcelina - HSM - São Paulo (SP), Brasil.

Os autores declaram não haver conflito de interesse na realização deste trabalho.

Não houve fonte pagadora ou patrocínio para realização deste trabalho.

Recebido em: 11/3/2010 Aceito em: 13/8/2010
} 
ram uma grave deformidade final: a biopsia percutânea foi realizada em 19 pacientes com positividade em 5 , não influenciando o tratamento do paciente. A dor pós-tratamento clínico apresentou melhora importante; foi utilizado esquema tríplice por um ano. Conclusão: o tratamento clínico da tuberculose deve ser iniciado assim que se suspeitar da doença e tiver imagens compatíveis com: corpo vertebral, diminuição da altura do espaço discal e elevação do ligamento longitudinal anterior. Na presença de cifose, o uso de um colete rígido deve ser ponderado, sendo ele o de Boston ou um colete gessado. A avaliação neurológica deve ser acompanhada com um intervalo curto, quinzenalmente nos primeiros três meses, pois se o tratamento clínico for ineficaz e o paciente apresentar déficit neurológico, o tratamento cirúrgico deve ser considerado. A biopsia é um exame de alta especificidade, mas de baixa sensibilidade. Quando positiva, reforça o tratamento medicamentoso. with positivity in 5 , with no influence patient treatment. The pain after treatment showed significant improvement and we used triple drug regimen for one year. Conclusions: the clinical treatment of tuberculosis should start once the disease is suspected and have compatible images with: vertebral body, decreased disc space height, and elevation of the anterior longitudinal ligament. In the presence of kyphosis using a weighted vest to be hard, being the Boston vest or a plaster cast. The neurological evaluation should be accompanied, and with a short interval, fortnightly during the first three months, because if the clinical treatment is ineffective and the patient has neurological deficit surgical treatment should be considered. The biopsy is a test of high specificity but low sensitivity. When the test is positive it reinforces drug treatment.

KEYWORDS: Spinal diseases; Biopsy; Tuberculosis, spinal/ therapy; Magnetic resonance imaging; Pain; kyphosis siendo los que presentaron una grave deformidad final; la biopsia percutánea fue realizada en 19 pacientes con positividad en 5, no influyendo en el tratamiento del paciente. El dolor post tratamiento clínico presentó una mejoría importante; fue utilizado un esquema triple por un año. Conclusión: el tratamiento clínico de tuberculosis debe ser iniciado así que la enfermedad fuera sospechada y tuviera imágenes compatibles con: el cuerpo vertebral, la disminución de la altura del espacio discal, y con la elevación del ligamento longitudinal anterior. En la presencia del cifosis, el uso de un chaleco rígido debe ser ponderado, siendo un Chaleco de Boston o un chaleco de yeso. La evaluación neurológica debe ser acompañada de un intervalo pequeño, quincenalmente en los primeros tres meses, pues si el tratamiento clínico fuera ineficaz y el paciente presentara déficit neurológico, el tratamiento quirúrgico debería ser considerado. La biopsia es un examen de alta especificidad, pero de baja sensibilidad. Si fuera positiva, refuerza el tratamiento medicamentoso.

DESCRIPTORES: Enfermedades de la columna vertebral; Biopsia; Tuberculosis de lacolumna vertebral/terapía; Imagen por resonancia magnética; Dolor; Cifosis

\section{INTRODUÇÃO}

A tuberculose é uma doença endêmica ${ }^{1}$ de natureza infecciosa causada pelo mycobacterium tuberculosis. Na literatura, existem relatos de múmias egípcias de 3.000 a.C. e manuscritos de Hippocrates 450 a.C. ${ }^{2}$.

Ela teve seu pico inicial na Revolução Industrial com os aglomerados populacionais. Dados atuais mostram que um terço da população mundial está infectada pela micobactéria e que oito milhões de novos casos são diagnosticados todo ano no mundo ${ }^{1}$. A localização pulmonar é a mais frequente, correspondendo a $95 \%$ dos casos registrados, sendo a forma inalatória a principal via de contaminação das formas extrapulmonares; cerca de $35 \%$ encontram-se no tecido músculo esquelético;
50 a $60 \%$ desses casos acometem a coluna; a grande maioria localiza-se na região toracolombar. Não se detecta o foco inicial em $31 \%$ dos casos diagnosticados na coluna ${ }^{1}$.

A disseminação da doença ocorre na circulação linfática, nos vasos sanguíneos ou por contiguidade. Na coluna, o plexo venoso de Batson é muitas vezes o responsável pela disseminação ${ }^{1}$.

As alterações vertebrais observadas nos exames de imagem surgem após cinco meses de instalação da doença óssea, e a via é a artéria nutrícia. A lesão do corpo vertebral pode produzir uma cifose. Na presença de material caseoso, pode ocorrer disseminação nos tecidos adjacentes - músculos e fascias, sendo esta a forma mais comum 
de acometimento do psoas e, algumas vezes, até mesmo do quadríceps ${ }^{1}$. A presença de instabilidade na coluna vertebral aumenta as chances de déficit neurológico. A clínica é composta de: dor localizada, rigidez, deformidade cifótica e déficit neurológico, o qual pode estar relacionado com o abscesso espondilítico. O desfecho após o início do tratamento medicamentoso consiste em cura do foco, lesão por fibrose, calcificação e anquilose. Os casos graves, resistentes aos tuberculostáticos, podem levar o paciente à morte ${ }^{1 .}$

\section{MÉTODOS}

Foi realizado estudo retrospectivo de maio de 1999 a junho de 2009, de abordagem quantitativa, no ambulatório de patologias da coluna vertebral do Hospital Santa Marcelina, São Paulo (SP).

Foram avaliados somente os pacientes que completaram todo o tratamento preconizado. Foram excluídos os pacientes que não fizeram o tratamento correto ou abandonaram o tratamento clínico. Todos os pacientes submeteram-se à investigação diagnóstica com exame radiográfico, tomografia computadorizada helicoidal (TC) e ressonância magnética (RM) da coluna, para avaliar o canal vertebral e/ou a presença de abscessos para vertebrais.

Para mensuração da dor foi utilizada a escala visual analógica de dor (EVA), com avaliação bimestral do item dor pelo período de um ano.

Para a avaliação neurológica aplicou-se quinzenalmente nos primeiros três meses, e depois a escala de Frankel.

O grau da cifose foi medido no exame radiográfico pelo Método de Cobb em todas as fases do tratamento e foi realizada uma comparação com as imagens iniciais.

A avaliação laboratorial constou de hemogramas seriados, acompanhados de análise dos valores da hemossedimentação (VSG) e proteína C (PCR).

$\mathrm{O}$ critério para diagnóstico clínico baseou-se no exame radiográfico do corpo vertebral, com diminuição da altura do espaço discal e elevação do ligamento longitudinal anterior, sendo o paciente submetido à TC (janela óssea) para definir melhor a destruição óssea e à RM, em que observamos o limite da coleção e a presença de sequestro. $\mathrm{O}$ uso de contraste mostrou um baixo sinal no interior da coleção caseosa ${ }^{3}$.

A indicação da biópsia ocorreu nos pacientes que apresentavam imagem radiológica atípica ou pouca melhora com o tratamento clínico, também associado a alterações na RM. Utilizou-se a técnica percutânea para biópsia com controle radioscópico em 19 pacientes. A indicação de intervenção cirúrgica foi reservada para dois pacientes com lesão na coluna dorsal e lombar, que evoluíram com déficit neurológico. Realizamos descompressão, pela abordagem posterior associada à costotransversectomia na torácica e posterolateral única na lombar, em um único tempo com melhora do status neurológico desses pacientes que necessitaram de biópsia aberta e instrumentação devido aos sinais de instabilidade com lesão neurológica. Nos dois casos relatados também foi solicitado anatomopatológico intraoperatório sem resultado conclusivo; nesses casos, um era na coluna torácica e outro na coluna lombar.

\section{RESULTADOS}

Foram encontrados 23 homens (74,2\%) e 8 mulheres (25,8\%). A média da idade foi de 48,9 anos (de 17 a 80, com desvio padrão de 14,93) (Figura 1).

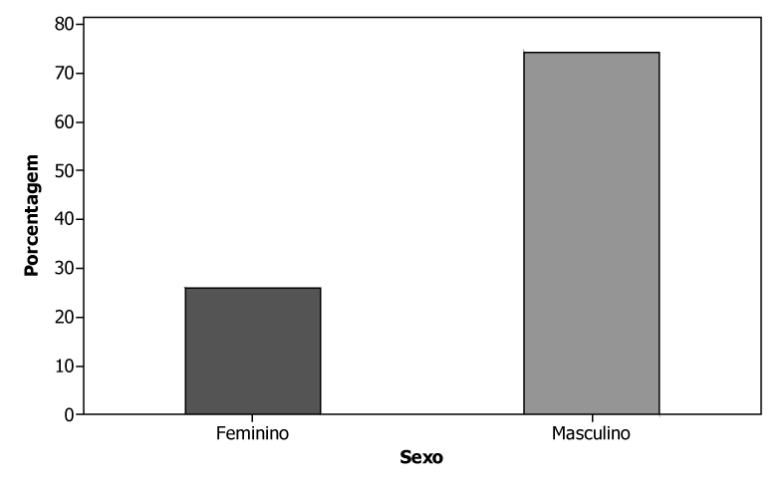

Figura 1

Relação da incidência por sexo $(p=0,007)$.

A cifose foi avaliada pelo método de Cobb, sendo padronizada a borda superior da vértebra saudável acima da lesão e a borda inferior da vértebra saudável abaixo da lesão. A cifose segmentar média foi de $49,95^{\circ}$ (de $17^{\circ}$ a $70^{\circ}$ ). A dor teve avaliação bimestral pela EVA, mostrando média de dor inicial 8,22 (de 6 a 10) com desvio padrão de 0,9. A dor pós-tratamento clínico mostrou 2,26 (de 0 a 5) com desvio padrão de 1,36, (Figura 2).



Figura 2

Dor entre o pré e o pós-tratamento (TT) $(p<0,001)$.

A biópsia foi feita em 21 pacientes (19 por agulha e 2 abertas) sendo positiva em 5 , correspondendo a $23,8 \%$ de positividade dos pacientes biopsiados e $16,5 \%$ de todos os pacientes. A negatividade foi observada em 16 casos, sendo $76,2 \%$ desses casos (Figura 3 ). 


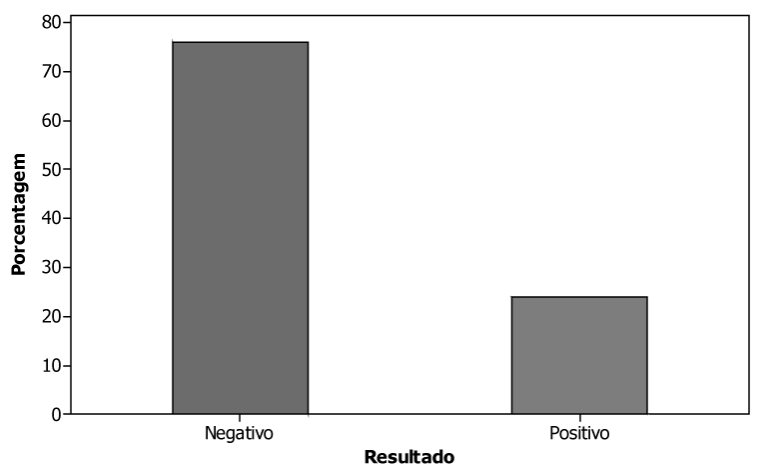

Figura 3

Resultado da biópsia $(p=0,016)$.

Os pacientes incluídos no estudo só apresentavam acometimento da coluna torácica ou lombar.

Encontramos 15 pacientes com lesão na coluna torácica $(48,4 \%)$ e 16 pacientes com lesão na coluna lombar $(51,6 \%)$ (teste qui-quadrado de aderência com $\mathrm{p}=0,857)$.

Foi observada coleção na RM em quatro pacientes, correspondendo a 12,9\% (teste qui-quadrado de aderência com $\mathrm{p}<0,001$ ), sendo necessário a drenagem em dois casos devido à piora da compressão e do déficit neurológico.

Observamos que nos dois pacientes submetidos à descompressão com instrumentação foi observada melhora neurológica em um paciente, que apresentava Frankel B, evoluindo para Frankel D após intervenção posterior. $\mathrm{O}$ outro paciente com quadro neurológico Frankel A não apresentou melhora após a cirurgia.

A melhora clínica e radiográfica ocorreu em 29 pacientes $(93,5 \%)$. Foi considerado melhora clínica o alívio da dor e os índices normais para o VSG e PCR. A melhora radiológica foi considerada quando ocorria a consolidação com anquilose do seguimento. Após diagnóstico e início do tratamento medicamentoso, os pacientes não apresentaram piora considerável da cifose inicial. O aumento da cifose ocorreu nos dois pacientes que apresentaram falha dos tuberculostáticos, com aumento de $10^{\circ}$ na coluna torácica e $7^{\circ}$ na coluna lombar. A medida da cifose final foi de $50,83^{\circ}$ (de $27^{\circ}$ a $70^{\circ}$ ). A melhora da dor baseada na EVA foi de 5,87 (com desvio padrão de 1,5).

Correlacionando as variáveis com o sexo, foram obtidos os seguintes resultados: o sexo feminino possui uma média de idade significativamente menor que o masculino (teste $t$-Student com $\mathrm{p}=0,024$ ) (Figura 4). Não encontramos diferenças significativas na distribuição de nível da lesão entre os sexos (teste qui-quadrado com $p=0,916$ ). Utilizando ANOVA de medidas repetidas, encontramos que ambos os sexos diminuíram significativamente o valor de EVA da dor $(\mathrm{p}<0,001)$, mas não encontramos diferenças entre os sexos nem no pré nem no pós-tratamento $(\mathrm{p}=0,182)$ (Figura 5).

O tratamento medicamentoso constou do esquema tríplice com rifampicina, pirazinamida e isoniazida pelo período de 12 meses.

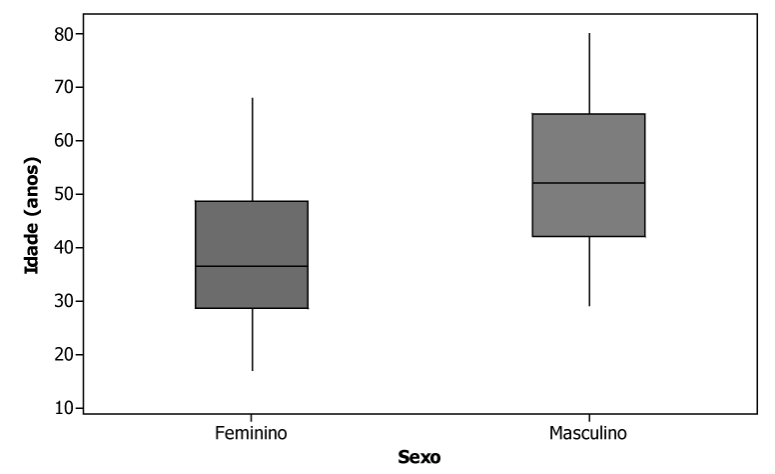

Figura 4

Relação do sexo por idade $(p=0,024)$.

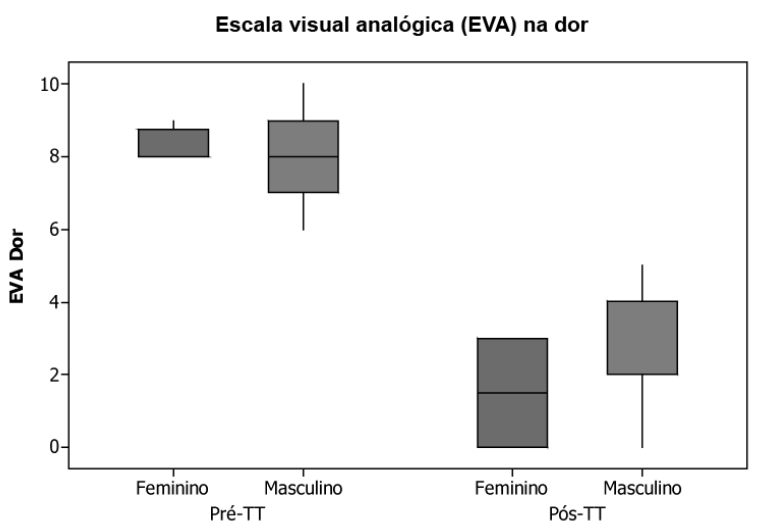

Figura 5

Avaliação da melhora da dor após tratamento clínico diferenciando entre os sexos $(p<0,001)$.

Colete rígido, de Boston ou gessado, foi utilizado em todos pacientes (Boston - 19 pacientes, colete gessado - 12).

\section{DISCUSSÃO}

A espondilite infecciosa é uma doença preocupante nos dias de hoje. O uso de RM facilitou o processo de diagnóstico precoce.

Este estudo retrospectivo avaliou os pacientes com tuberculose acompanhada em nosso serviço, em conjunto com o grupo da tisiologia, que fez o suporte clínico.

Apoiados na literatura mundial, optamos por seguir a conduta, quanto à indicação da cirurgia, divergindo apenas quanto à realização da biópsia ${ }^{1,2,4}$, que foi feita com agulha em 19 e aberta em 2; com positividade em apenas 5 (Figura 6).

Em seu trabalho, Abhay Nene ${ }^{5}$ discute o tratamento cirúrgico, indicando-o apenas quando o paciente apresenta Frankel A ou B. O tratamento clínico tem bons resultados segundo esses autores, os pacientes operados apresentaram melhora do nível neurológico, fato observado em nosso estudo. Kalita ${ }^{6}$ apresenta casos em que após seis meses de pós-operatório os pacientes estavam recuperados do déficit neurológico. Benli et al. $^{3}$ apresentam dados estatísticos diferentes, sendo a coluna torácica mais acometida, e cifose 


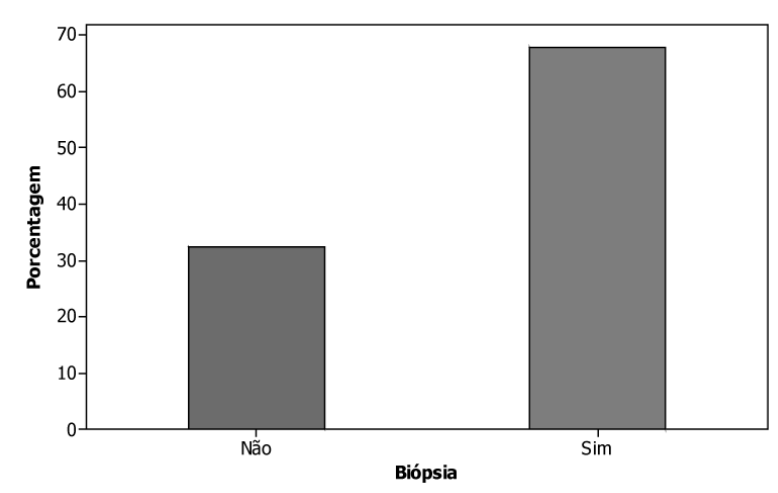

Figura 6

Pacientes que foram submetidos à biópsia $(p=0,048)$.

residual média com valores inferiores ao grupo estudado, com uma média de $23^{\circ}$, apresenta resultados satisfatórios com desbridamento e instrumentação anterior, enquanto este trabalho obteve boa evolução com tratamento clínico.

$\mathrm{Chang}^{7}$, em seu trabalho, relata que as imagens da tuberculose na RM são específicas, observando a destruição óssea, com preservação do disco na maioria dos casos. $\mathrm{Na}$ falha do tratamento ocorre destruição do corpo vertebral muito rápido, fato este que observamos nos pacientes que chegaram com um tempo maior de evolução do quadro, em que a perda óssea foi importante.

Encontramos, no nível lombar, uma média de cifose significativamente menor que no nível torácico (teste $t$ Student com $\mathrm{p}<0,001)$.

Puertas $^{8}$, em seu trabalho, descreve o tratamento de 17 pacientes, tendo indicado cirurgia em três deles, dando ênfase ao tratamento clínico com bons resultados, fato observado também em nosso estudo. Utilizando ANOVA de medidas repetidas, encontramos que, para quem realizou ou não biópsia, houve diminuição significativa do valor da EVA ( $p<0,001)$, mas não encontramos diferenças entre aqueles que realizaram ou não biópsia, nem no pré nem no pós-tratamento ( $\mathrm{p}=0,700)$ (Figura 7$)$.

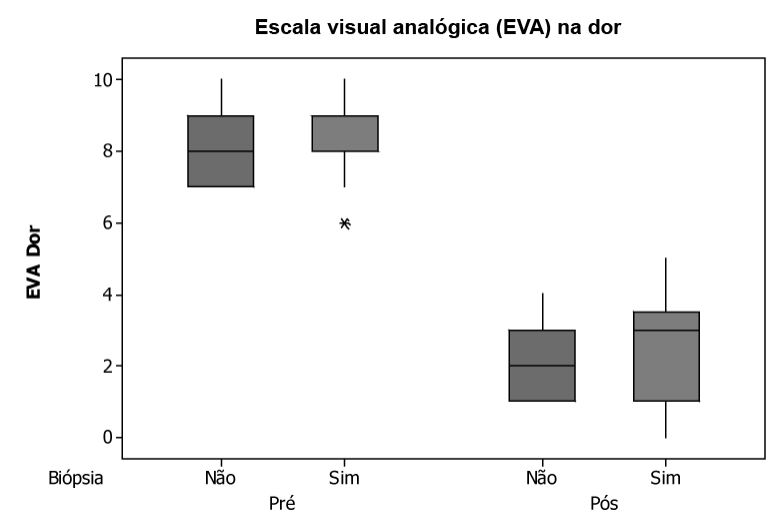

Figura 7

Resultado mostrando melhora clínica independente da biópsia $(p<0,001)$.
Em um estudo recente realizado em Goiânia ${ }^{9}$, os pesquisadores relataram a pouca influência da biópsia no tratamento e prognóstico dos pacientes. A terapêutica com esquema tríplice era instituída com a suspeita da doença. Em nosso estudo também observamos esse fato; a biópsia percutânea ou aberta teve pouca influência no prognóstico dos paciente (Figuras de 8 a 11). Luk ${ }^{10}$ relata bons resultados com tratamento clínico, porém no seu follow-up de 15 anos, ele observou que a cifose pode progredir, fato não observado por nós, apesar de nosso maior período de seguimento ser um caso com 11 anos de evolução. Encontramos que o grupo com presença de abscesso possui uma média de cifose significativamente maior do que o grupo que não possui coleção (teste $t$-Student com p=0,017) (Figura 12).
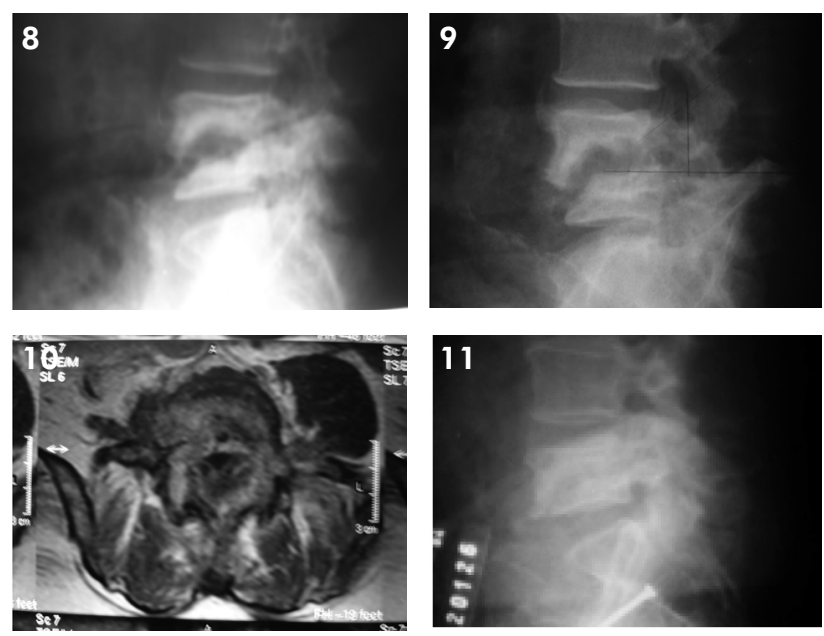

Figuras 8, 9, 10 e 11

Controle inicial e anquilose óssea IRM com cicatriz em partes moles e corpo vertebral).

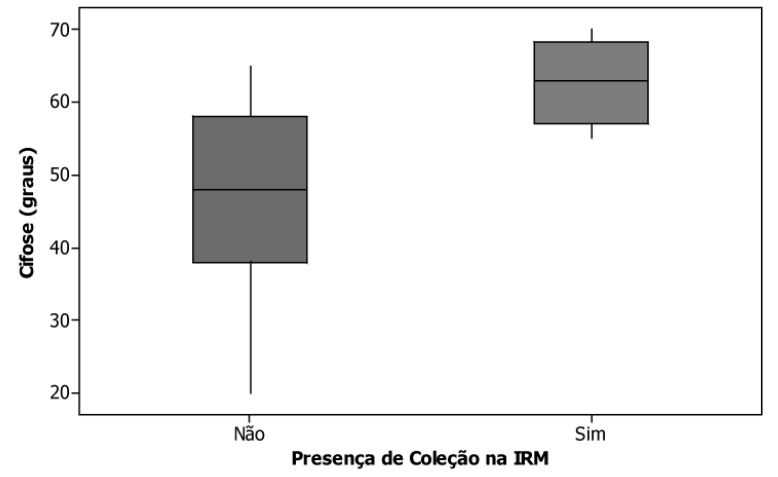

Figura 12

Relação da cifose com relação a coleção mostrando uma piora angular nos pacientes com coleção $(p=0,017)$.

\section{CONCLUSÃO}

A espondilodiscite tuberculosa deve sempre ser pensada em pacientes que vivem em áreas conglomeradas rural ou urbana. O tratamento clínico deve ser iniciado assim que se suspeite da doença, e os critérios de imagem 
forem compatíveis. Na possibilidade de cifose, o uso de um colete rígido deve ser ponderado. A avaliação neurológica deve ser acompanhada com um intervalo pequeno, pois, se o tratamento clínico for ineficaz e o paciente apresentar déficit, o tratamento cirúrgico deve ser considerado.
A tuberculose da coluna vertebral diagnosticada precocemente apresenta boa evolução com tratamento clínico na maioria dos casos.

A biópsia é um exame de alta especificidade, mas de baixa sensibilidade, não devendo ser descartada; porém, não devemos basear o tratamento dos pacientes em sua negatividade.

\section{REFERÊNCIAS}

1. Frías Salcedo JA, Gutiérrez Bautista AE, Santiago Tipac G. Tuberculosis vertebral multiple: reporte de un caso y revisión de la literatura. Rev Mex Ortop Traumatol. 2000;14(4)360-5.

2. Griffith JF, Kumta SM, Leung PC, Cheng JC, Chow LT, Metreweli C. Imaging of musculoskeletal tuberculosis: a new look at an old disease. Clin Orthop. 2002;(398):32-9.

3. Benli IT, Acaroğlu E, Akalin S, Kis M, Duman E, Un A. Anterior radical debridement and anterior instrumentation in tuberculosis spondylitis. Eur Spine J. 2003;12(2):224-34.

4. Branco Castyello BP, Souza AB. Espondilodiscite tuberculosa. J Bras Med. 2001;80(6): 37-45.

5. Nene A, Bhojraj S. Results of nonsurgical treatment of thoracic spinal tuberculosis in adults. Spine J. 2005;5(1):79-84.
6. Kalita J, Misra UK, Mandal SK, Srivastava M. Prognosis of conservatively treated patients with Pott's paraplegia: logistic regression analysis: J Neurol Neurosurg Psychiatry. 2005;76(6):866-8.

7. Chang MC, Wu HT, Lee CH, Liu CL, Chen TH. Tuberculous spondylitis and pyogenic spondylitis: comparative magnetic resonance imaging features. Spine (Phila Pa 1976). 2006;31(7):782-8.

8. Puertas EB, Chagas JCM, Wajchenberg M, D'Orto CCC. Avaliação clínica, radiológica e tratamento de 17 pacientes com tuberculose óssea na coluna vertebral. Rev Bras Ortop. 1999;34(2):113-6.

9. Daher S, Cardoso ALP, Souza Júnior ZA, Pimenta Júnior WE, Moraes FB, Daher MT et al. Tuberculose espinhal: avaliação de 26 casos. Coluna/ Columna. 2006;5(1):1-6.
10.Luk KD. Tuberculosis of the spine in the new millennium. Eur Spine J. 1999;8(5):338-45.

\section{Correspondência}

Luiz Claudio Lacerda Rodrigues

Hospital Santa Marcelina

Setor de Ortopedia e Traumatologia

Rua Santa Marcelina, 177 - Itaquera

CEP 08270-070 - São Paulo (SP), Brasil

Fone: 2170-6000 ramal 6317

E-mail: luizclr@terra.com.br 